Article

\title{
In Vitro Cytotoxic Protective Effect of Alginate-Encapsulated Capsaicin Might Improve Skin Side Effects Associated with the Topical Application of Capsaicin
}

\author{
Ariana Hudita ${ }^{1} \mathbb{D}$, Bianca Galateanu ${ }^{1} \mathbb{D}$, Marieta Costache ${ }^{1} \mathbb{D}$, Carolina Negrei ${ }^{2}{ }^{*}$, Rodica-Mariana Ion ${ }^{3}(\mathbb{D}$, \\ Lorena Iancu ${ }^{3}$ and Octav Ginghina ${ }^{4,5}$
}

1 Department of Biochemistry and Molecular Biology, University of Bucharest, 050095 Bucharest, Romania; arianahudita@yahoo.com (A.H.); Bianca.galateanu@bio.unibuc.ro (B.G.);

Marieta.costache@bio.unibuc.ro (M.C.)

2 Department of Toxicology, Faculty of Pharmacy, "Carol Davila” University of Medicine and Pharmacy, 020021 Bucharest, Romania

3 National Institute of Research and Development for Chemistry and Petrochemistry-ICECHIM, 060021 Bucharest, Romania; rodica.ion@icechim.ro (R.-M.I.); lorena.iancu@icechim.ro (L.I.)

4 Department of Surgery, "Sf. Ioan" Clinical Emergency Hospital, 042122 Bucharest, Romania; octavginghina@yahoo.com

5 Department II, Faculty of Dental Medicine, "Carol Davila” University of Medicine and Pharmacy, 020021 Bucharest, Romania

* Correspondence: cnegrei@gmail.com

\section{check for} updates

Citation: Hudita, A.; Galateanu, B.; Costache, M.; Negrei, C.; Ion, R.-M.; Iancu, L.; Ginghina, O. In Vitro Cytotoxic Protective Effect of Alginate-Encapsulated Capsaicin Might Improve Skin Side Effects Associated with the Topical Application of Capsaicin. Molecules 2021, 26, 1455. https://doi.org/ 10.3390/molecules26051455

Academic Editor: Piyali Dasgupta

Received: 4 February 2021

Accepted: 2 March 2021

Published: 7 March 2021

Publisher's Note: MDPI stays neutral with regard to jurisdictional claims in published maps and institutional affiliations.

Copyright: (C) 2021 by the authors. Licensee MDPI, Basel, Switzerland. This article is an open access article distributed under the terms and conditions of the Creative Commons Attribution (CC BY) license (https:/ / creativecommons.org/licenses/by/ $4.0 /)$.

\begin{abstract}
Chronic neuropathic pain, particularly peripheral pain, is a cause of great concern for diabetic patients. Current treatments include numerous agents such as capsaicinoids, a known deterrent of neuropathic pain despite the inconvenience associated with local side effects. In this context, the current work aims to elucidate the potential mechanisms involved in cytotoxicity by capsaicin and proposes an efficient formulation of capsaicin in alginate microcapsules, which significantly reduces side effects from capsaicin topical administration. For this, human dermal fibroblast cells were treated with alginate-microencapsulated capsaicin extracts and screened for potential cytotoxic effects produced by the treatment. Cell viability and morphology were examined, as well as oxidative stress status and anti-inflammatory potential. Our results show that the alginate encapsulated formulation of capsaicin exerted lower cytotoxic effects on human dermal fibroblasts as measured by cell viability and reactive oxygen species (ROS) production. Furthermore, the expression profiles of inflammatory cytokines were significantly altered by the treatment as compared with the control culture.
\end{abstract}

Keywords: capsaicin; alginate encapsulation; neuropathic pain; cytotoxicity; inflammation

\section{Introduction}

The use of capsaicinoids for the treatment of pain goes as far back as $4000 \mathrm{BC}$ [1] However, it was only introduced to the Western world in the 15th century, on Columbus' return to Europe from his successful discovery trip to the Americas, and chilies were one of the wonder products he brought over. First only used as a very attractive spice, the medicinal powers of chilies (and capsaicinoids) were discovered much later in the middle of the 19th century, when their peculiar capacity to alleviate pain by topical application turned them into a very appreciated remedy against burns or itches in the extremities [2]. Also known as "green peppers", this fruit's name is rather improper, since they are a member of the genus Capsicum. They owe their hot and pungent taste not so much to capsaicin as to piperines, the usual and defining component of the Piperaceae family [3]. From over 20 known major non-endogenous capsaicinoids, most (as much as 90\%) naturally occurring ones are capsaicin and dihydrocapsaicin [4]). Others include homodihydrocapsaicin, nordihydrocapsaicin, and homocapsaicin [4]. 
As a result of continued research, further medicinal properties of capsaicinoids were discovered, and they are currently studied for their potential as analgesics, antioxidants, anticarcinogens [5], and pharmacological agents against obesity [4,6-8].

Capsaicin intended for trade purposes is an oily extract, authorized as a cream or patch, which is used in the treatment of chronic pain syndromes as postherpetic neuralgia, musculoskeletal pain, arthrosis, rheumatoid arthritis, rash, psoriasis, bladder conditions (neurogenic bladder), and, last but not least, diabetic neuropathy [9,10]. Capsaicin (trans-8methyl- $N$-vanillyl-6-nonenamide), known as chili pepper fruit, is a natural alkaloid derived from plants of the genus Capsicum. Like other vanilloids, capsaicin has a benzene ring and a long hydrophobic carbon tail with a polar amide group. Capsaicin is not water-soluble, and for its solubilization, different alcohols and other organic solvents are used in topical preparations and sprays. In order to keep capsaicin stable for a long time and to increase its solubility, this compound has been encapsulated by different biomaterials, such as alginate, which is recognized for its low toxicity. From a chemical point of view, alginate is a linear copolymer containing blocks of $(1,4)$-linked $\beta$-D-mannuronate and $\alpha$-L-guluronate residues. Alginate is useful as a matrix for cell immobilization, as well as for the entrapment of bioactive compounds and drugs. Encapsulated drugs are released from alginate pellets by diffusional processes through pores in the polymeric network [11].

Chronic neuropathic pain, particularly peripheral pain related to diabetes, is a cause for concern for several reasons, including the worsening of pain at night, which causes sleep deprivation and an entire range of related subsequent effects such as fatigue, poor performance, and poor social integration.

Treatment is typically aimed at pain modulation, patient education regarding pain management, and restoration of motor function, all relying on constant and careful glycemic control [11]. The treatments available have been limited by adverse reactions, leading to suboptimal benefit/risk ratio. Furthermore, among the numerous agents researched, there has been renewed interest in finding further means to use the analgesic action of capsaicinoids as a deterrent of neuropathic pain, resulting in the emergence on the market and pharmaceutical development of a variety of capsaicin-containing products. These products include quasi-traditional OTC capsaicin preparations and low $(<1.0 \%)$ concentration capsaicin. Such efforts were proven necessary by the comparatively unsatisfactory efficacy of existing products, which has been aggravated by poor patient compliance arising from the need for multiple topical applications over extended periods as a result of insufficient effectiveness. Among the solutions proposed and tested, the development of products with increased capsaicin strength, i.e., the capsaicin $8 \%$ patch, have shown promising potential to alleviate pain by a single topical application.

In regard to the management of diabetic neuropathic peripheral pain, studies suggest that capsaicin may be effective to a certain degree, but its use is fraught with a frustrating number of limitations. Inconveniences such as an unpleasant burning sensation on initial application, the extended time needed for sufficient depletion of pain, the necessity for multiple applications to maintain analgesic efficacy, problematic effects in case of discontinuation for longer than $24 \mathrm{~h}$, and compelling sustained capsaicin application for substance $\mathrm{P}$ replenishing all inflict upon the potential for the widespread use of current capsaicin pharmaceutical formulations, aggravating the need to develop new formulations of enhanced efficacy and limited adverse effects [12,13].

In this context, this work aims to elucidate the potential mechanisms involved in the cytotoxicity of capsaicin and proposes an efficient formulation of capsaicin in alginate microcapsules, which significantly reduces the side effects of the topical administration of capsaicin.

\section{Results and Discussion}

\subsection{Alginate Microcapsules and Capsaicin Encapsulation}

The encapsulation efficiency (EE, \%) is a variable that allows us to quantify the amount of active principle (capsaicin, in this case) that is encapsulated and can be calculated by 
analyzing the amount that has not been encapsulated or the amount that is lost during the microparticle consolidation stage. Quantification was carried out by UV-vis. Calcium alginate spherical microcapsules, shown in Figure 1, were prepared by dropping a sodium alginate solution containing a suspension of the gelatin microparticles into a $\mathrm{CaCl}_{2}$ solution .

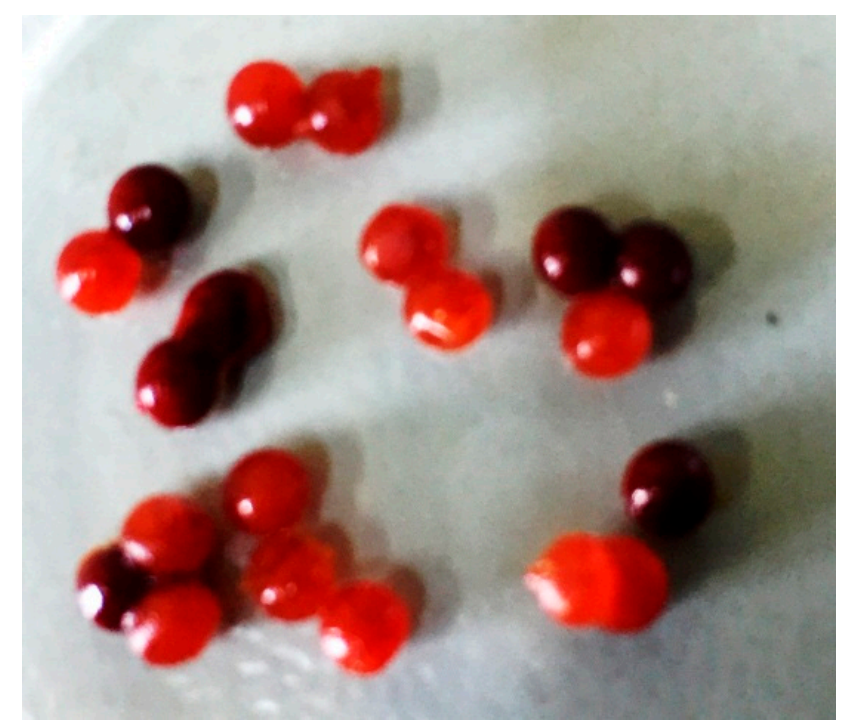

Figure 1. Microscopy image of alginate microcapsules loaded with capsaicin.

The encapsulation efficiency of capsaicin can be expressed by the Equation (1), presented in the Materials and Methods section. For these experiments, by applying the above-mentioned formula, the obtained encapsulation efficiency was $87 \%$.

\subsection{In Vitro Cytotoxic Activity of Capsaicin-Loaded Alginate Microcapsules}

The viability of CCD-1070Sk dermal fibroblasts was investigated after $24 \mathrm{~h}$ of treatment with the collected extracts and different concentrations of free capsaicin. Cellular mitochondrial activity was analyzed using the MTT quantitative assay. The obtained data were statistically analyzed using GraphPad Prism software and are graphically represented in Figure 2. The viability of dermal fibroblasts exposed to free capsaicin decreased significantly after $24 \mathrm{~h}$ of treatment as compared to that of the untreated samples, regardless of the concentration of capsaicin used. In comparison, extracts of AM (alginate microcapsules) and AM loaded with capsaicin, collected at $30 \mathrm{~min}$ and $3 \mathrm{~h}$, did not show any significant effect on CCD-1070Sk cell viability after $24 \mathrm{~h}$ of treatment as compared with the untreated samples. Furthermore, CCD-1070Sk cells exposed to capsaicin loaded AM extracts for $24 \mathrm{~h}$ had a statistically significant increase in cellular viability for all concentrations of capsaicin, demonstrating that the microencapsulation of capsaicin alleviates its cytotoxicity.

To further explore the cytotoxicity of the collected extracts and different concentrations of free capsaicin, the release of lactate dehydrogenase (LDH) in culture cell media was measured after $24 \mathrm{~h}$ of treatment. As shown in Figure 3, a statistically significant increase in LDH release was observed in response to $24 \mathrm{~h}$ exposure of CCD-1070Sk cells to free capsaicin solutions for all screened concentrations, highlighting the cytotoxic potential of free capsaicin on human dermal fibroblasts. Furthermore, the cytotoxic profile of the extracts collected from unloaded AM revealed that the bare micro formulation did not trigger any alteration in the LDH activity after $24 \mathrm{~h}$ of treatment. Also, the same cytotoxic profile was noticed for the extracts collected from the capsaicin-loaded AM for all concentrations tested, showing that the microencapsulation of capsaicin represents a strong tool for modulating free capsaicin cytotoxicity. 


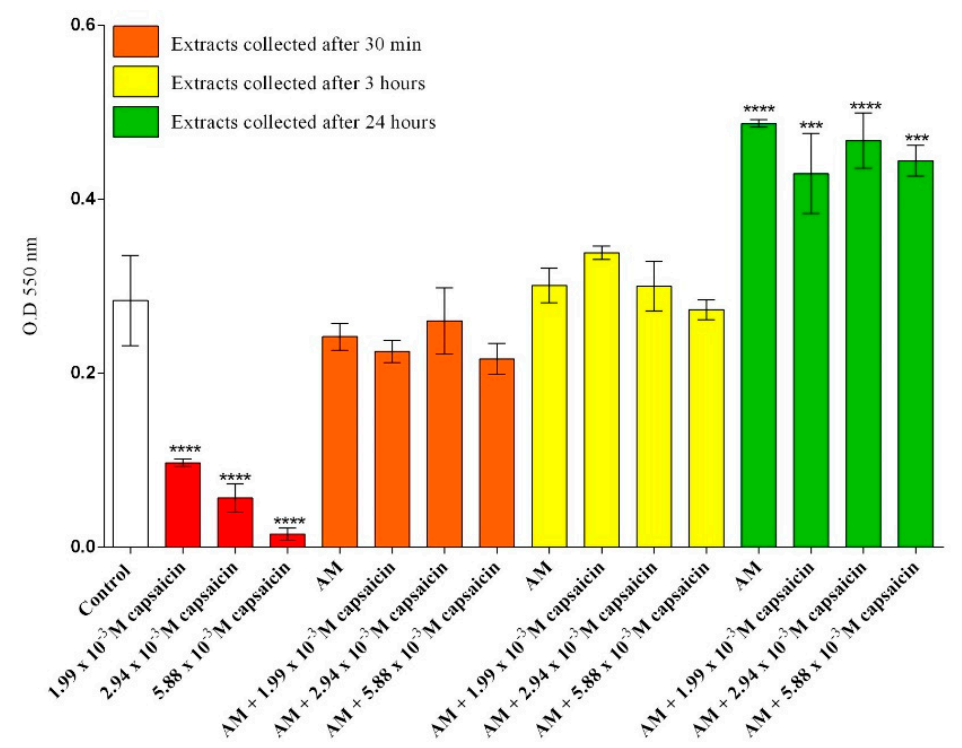

Figure 2. Measurement of cell viability after $24 \mathrm{~h}$ of exposure to different treatments as revealed by MTT assay ${ }^{* * *} p \leq 0.001$ treated vs. control; ${ }^{* * * *} p \leq 0.0001$ treated vs. control).

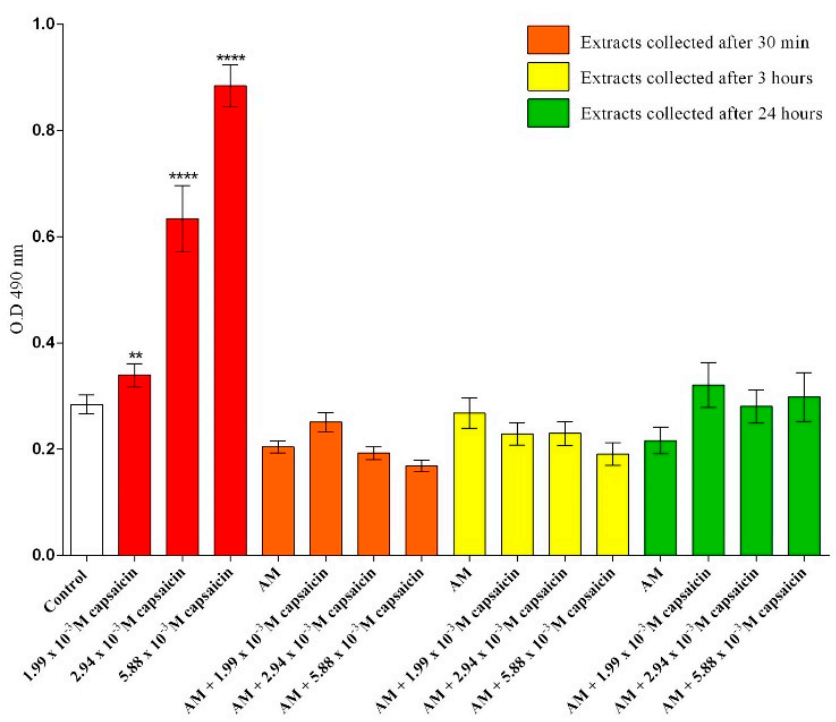

Figure 3. Quantification of LDH release after $24 \mathrm{~h}$ of exposure to different experimental conditions ${ }^{* *} p \leq 0.01$ treated vs. control; ${ }^{* * *} p \leq 0.0001$ treated vs. control).

In order to obtain an overview of the potential cytotoxic effects of free and microencapsulated capsaicin on human dermal fibroblasts, cellular viability was evaluated by labelling both live and dead cells using a Live/Dead assay. The images captured by fluorescence microscopy investigation of the samples are presented in Figure 4. Our results show that all concentrations of free capsaicin dramatically alter cellular viability, since almost exclusively dead cells are scattered on the culture surface after the exposure of CCD-1070Sk cells to treatment. However, no dead cells were detected in CCD-1070Sk cell cultures exposed to $24 \mathrm{~h}$-collected extracts, showing that neither simple AM nor drug-loaded AM trigger cell death in human dermal fibroblasts cells in vitro. The ratio and distribution of live cells on the cell culture surface in CCD-1070Sk cells exposed to the tested extracts for $24 \mathrm{~h}$ are similar to the control cell culture, showing that both bare and loaded AM lack cytotoxic potential towards human dermal fibroblasts. 
CONTROL

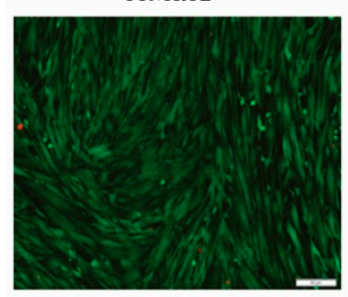

AM

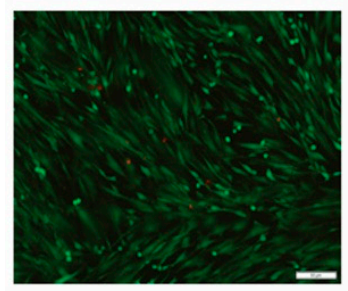

$1.99 \times 10^{3}$ M Capsaicin

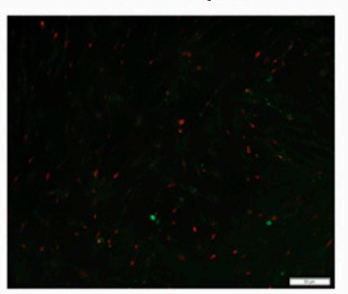

$\mathrm{AM}+1.99 \times 10^{-3} \mathrm{M}$ capsaicin

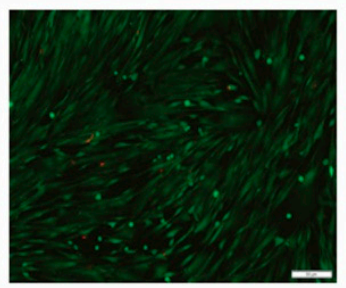

$2.94 \times 10^{3} \mathrm{M}$ Capsaicin

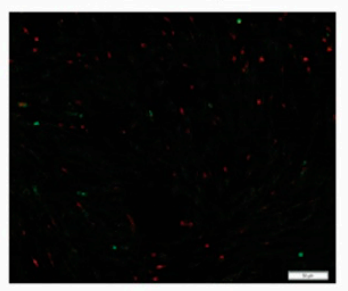

$\mathrm{AM}+2.94 \times 10^{-3} \mathrm{M}$ capsaicin

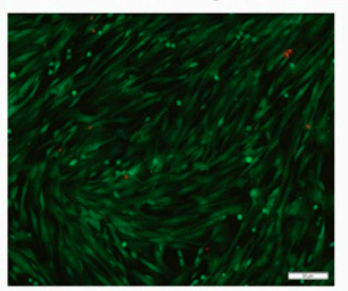

$5.88 \times 10^{3}$ M Capsaicin

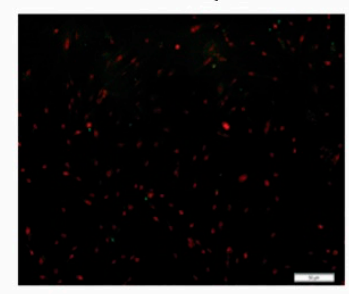

$\mathrm{AM}+5.88 \times 10^{-3} \mathrm{M}$ capsaicin

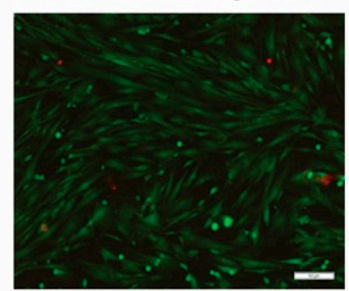

Figure 4. Fluorescence micrographs of live/dead stained CCD-1070Sk cells after 24 h exposure to different solutions of capsaicin and various extracts collected from simple AM or capsaicin-loaded AM (green fluorescence: live cells; red fluorescence: dead cells).

The results revealed that the novel microformulation loaded with capsaicin presents no significant cytotoxicity, irrespective of the loaded concentration of capsaicin. Therefore, we selected the microformulation loaded with the highest concentration of capsaicin, $5.88 \times 10^{-3} \mathrm{M}$, for further studies; in this view, extracts collected from $5.88 \times 10^{-3} \mathrm{M}$ capsaicin AM after $24 \mathrm{~h}$ were further used as cell treatments.

\subsection{CCD-1070Sk Cell Morphology after Capsaicin-Loaded AM Treatment}

In order to investigate the potential differences in cellular morphology between human dermal cells treated with capsaicin or capsaicin-loaded AM ( $24 \mathrm{~h}$ extracts), we investigated the cytoskeleton actin filaments after FITC-phalloidin staining. As shown in Figure 5, a low number of CCD-1070Sk cells were still adhered to the culture surface after $24 \mathrm{~h}$ of treatment with $5.88 \times 10^{-3} \mathrm{M}$ capsaicin. Moreover, the few adhered cells presented with a polygonlike shape and failed to develop long actin filaments. Regarding simple or capsaicin-loaded AM treatments, it is clearly shown that none of the treatments adversely affected the global morphology of human dermal fibroblasts. After $24 \mathrm{~h}$ of treatment, CCD-1070Sk cells showed a typical spindle-like shape and a well-developed cytoskeleton characterized by long actin filaments. Interestingly, CCD-1070Sk cells treated with capsaicin-loaded AM presented with a better developed actin filament network compared to cells treated with pristine AM. These results suggest that microencapsulated capsaicin stimulates actin protein expression and promotes intercellular contacts.

\subsection{Oxidative Stress Induced by Capsaicin-Loaded AM Treatment in CCD-1070Sk Cells}

In order to evaluate the potential of capsaicin-loaded AM (24 h extracts) to induce reactive oxygen species (ROS), the production of $\mathrm{H}_{2} \mathrm{O}_{2}$ was quantified as an indicator of oxidative stress using a ROS-Glo $\mathrm{H}_{2} \mathrm{O}_{2}$ assay. The obtained data are graphically represented in Figure 6 and revealed that free capsaicin induces ROS generation, as significantly enhanced levels of $\mathrm{H}_{2} \mathrm{O}_{2}$ were detected in treated CCD-1070Sk cell cultures compared to control cultures. Moreover, free capsaicin induced a rapid generation of hydrogen peroxide, as a significant increase in $\mathrm{H}_{2} \mathrm{O}_{2}$ levels was detected even after $6 \mathrm{~h}$ of treatment. In contrast, alginate microencapsulated capsaicin suppressed ROS production as no modifications to $\mathrm{H}_{2} \mathrm{O}_{2}$ levels were observed in AM and capsaicin-AM treated CCD-1070Sk cells compared to control cultures. Therefore, we conclude that alginate microcapsules and capsaicin-loaded microcapsules lack the ability to trigger $\mathrm{H}_{2} \mathrm{O}_{2}$ production in CCD-1070Sk cell cultures, as both treatments did not augment $\mathrm{H}_{2} \mathrm{O}_{2}$ levels after $6 \mathrm{~h}$ or $24 \mathrm{~h}$ of treatment. 


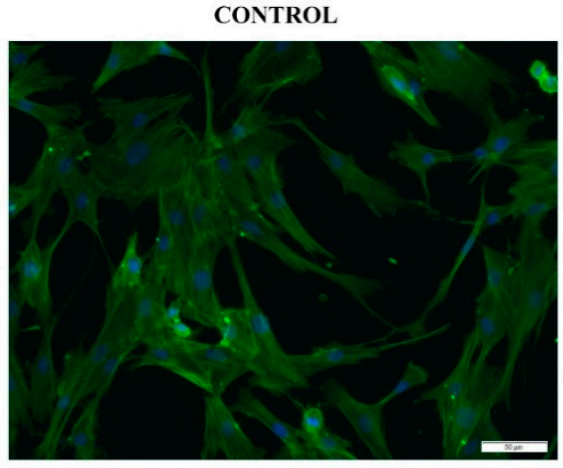

$\mathrm{AM}+5.88 \times 10^{-3} \mathrm{M}$ capsaicin

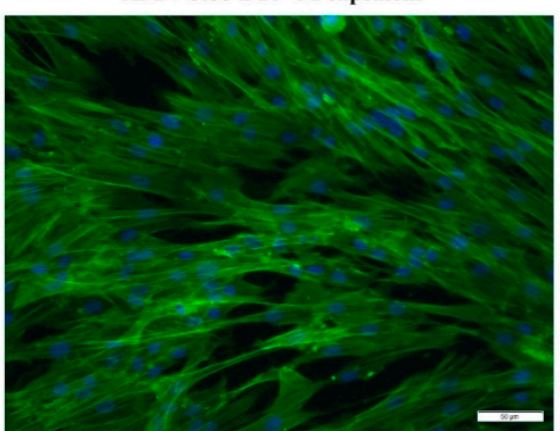

AM

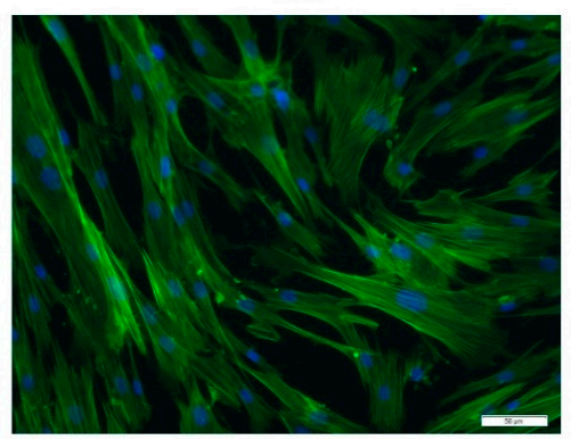

$5.88 \times 10^{-3}$ M Capsaicin

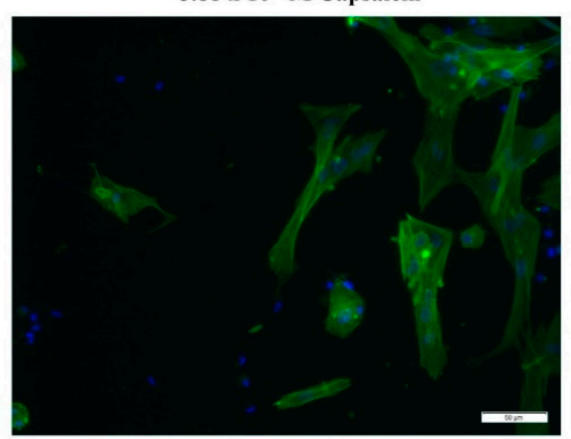

Figure 5. CCD-1070Sk cell morphology after $24 \mathrm{~h}$ of exposure to $5.88 \times 10^{-3} \mathrm{M}$ capsaicin, AM extract, and $5.88 \times 10^{-3} \mathrm{M}$ capsaicin AM extract as revealed by phalloidin staining of actin filaments (green). Cellular nuclei are stained with 2-(4-amidinophenyl)-1H-indole-6-carboxamidine (DAPI) (blue).

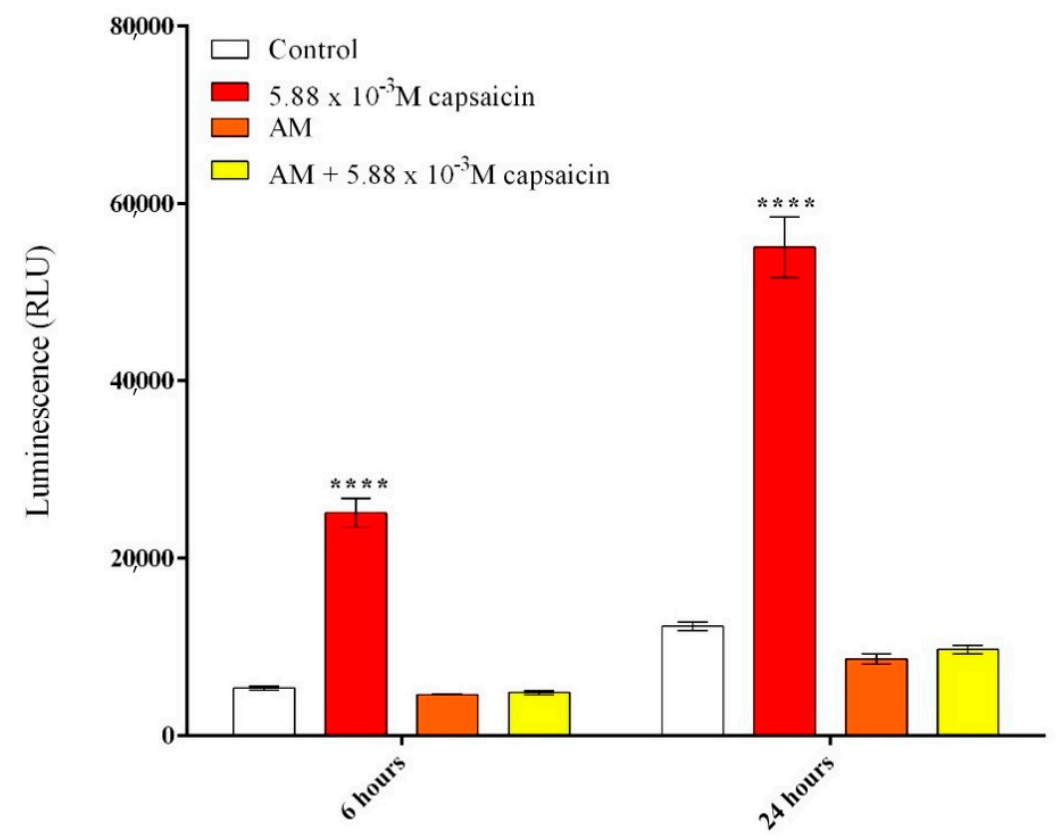

Figure 6. Effects of capsaicin and microencapsulated capsaicin treatment on ROS production after $6 \mathrm{~h}$ and $24 \mathrm{~h}$ of treatment in CCD-1070Sk cells as revealed by the ROS-Glo $\mathrm{H}_{2} \mathrm{O}_{2}$ assay. ( ${ }^{* * * *} p \leq 0.001$ treated vs. control).

Lipopolysaccharide (LPS) from E. coli was used to stimulate RAW 264.7 macrophages to produce NO. Griess reagent was used to determine NO production as an indicator of oxidative stress. The obtained data are graphically represented in Figure 7 and revealed that free capsaicin significantly increased NO generation even after $1 \mathrm{~h}$ of exposure compared 
to stimulated RAW cells. Capsaicin-loaded AM (24 h extracts), as well as unloaded AM ( $24 \mathrm{~h}$ extracts), did not alter the production of NO in the stimulated cells during the first $6 \mathrm{~h}$ of the experiment. However, after $24 \mathrm{~h}$, capsaicin-loaded AM (24 h extracts) significantly decreased NO production compared to the control.

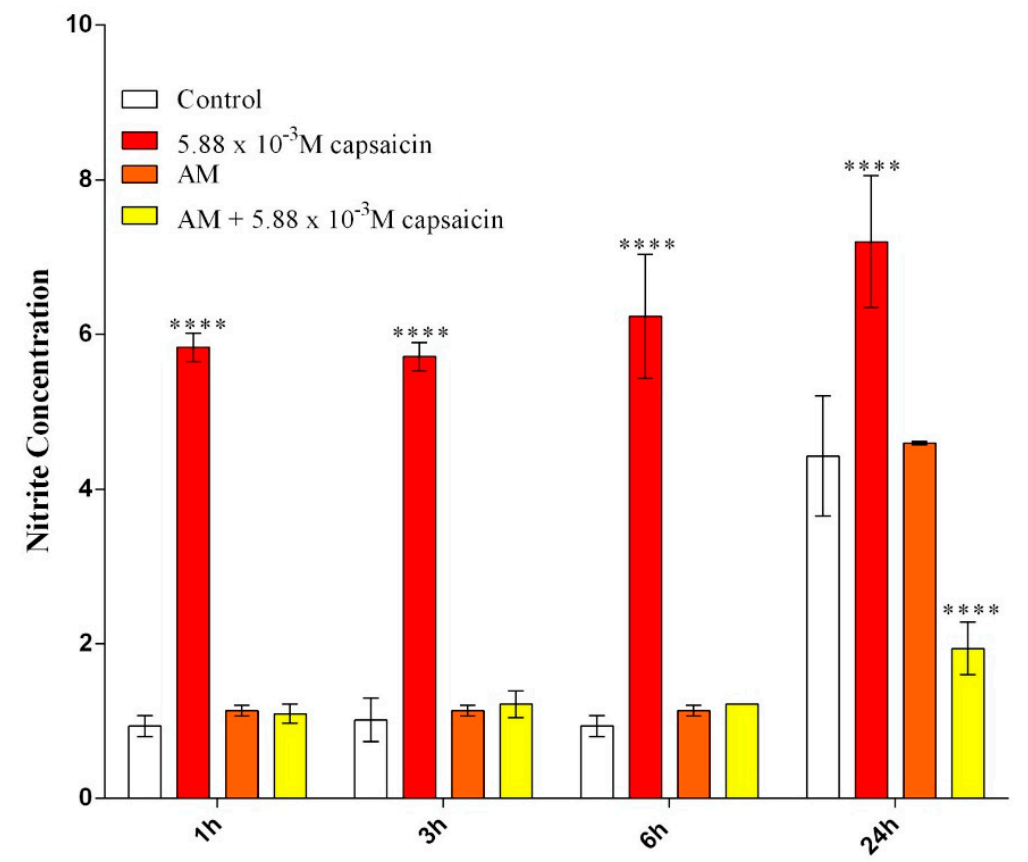

Figure 7. Effects of capsaicin and microencapsulated capsaicin treatment on NO production after $1 \mathrm{~h}$, $3 \mathrm{~h}, 6 \mathrm{~h}$, and $24 \mathrm{~h}$ of treatment in LPS-stimulated RAW 264.7 cells as revealed by Griess reagent assay ${ }^{* * * *} p \leq 0.0001$ treated vs. control).

\subsection{Evaluation of the Anti-Inflammatory Activity of Microencapsulated Capsaicin}

Lipopolysaccharide (LPS) from E. coli was used to stimulate RAW 264.7 cells to produce cytokines. The expression of IL-1 $\beta$, IL-6, IL-10, IL-12p70, MCP-1, MIP-1 $\alpha$, RANTES, and TNF- $\alpha$ were determined after $3 \mathrm{~h}$ and $24 \mathrm{~h}$ of LPS stimulated RAW 264.7 cell exposure to free capsaicin and capsaicin-loaded AM (24 h extracts) using a custom Mouse Cytokine/Chemokine Magnetic Bead Panel 96-well plate assay (MCYTOMAG-70k, Merck Millipore). The results are presented in Figure 8 and show that the treatments had a significant impact, starting from $3 \mathrm{~h}$ of exposure for many cytokines. More specifically, the expression of IL-1 $\beta$, a pro-inflammatory cytokine involved in pain [14], was significantly decreased after $3 \mathrm{~h}$ and $24 \mathrm{~h}$ of treatment with capsaicin-loaded AM compared to the untreated LPS stimulated cells. IL-6 is an inflammatory cytokine responsible for regulating a wide-range of biological pathways, including the development of acute pathological pain [15]. Our results show that the expression of IL-6 was significantly decreased after $3 \mathrm{~h}$ of treatment with free capsaicin, unloaded AM, and capsaicin-loaded AM extracts. However, after $24 \mathrm{~h}$ of treatment with free capsaicin, IL-6 levels were found to be significantly increased, while treatment with encapsulated capsaicin revealed a significand decrease in IL-6 expression. Similar patterns of expression were observed for MCP-1, MIP-1 $\alpha$, RANTES, and TNF- $\alpha$. Raised levels of MCP-1 have been observed in patients with chronic muscle pain, while direct evidence for its role as an algogen is still lacking. MIP-1 $\alpha$ mediates the development of neuropathic pain following peripheral nerve injury through IL-1 $\beta$ upregulation [16]. RANTES is a pro-inflammatory chemokine that directly interacts with opioid receptors and modifies the nociceptive reaction [17]. No significant modifications were observed with respect to the expression of IL-10 and IL-12p70 anti-inflammatory cytokines with these treatments. 

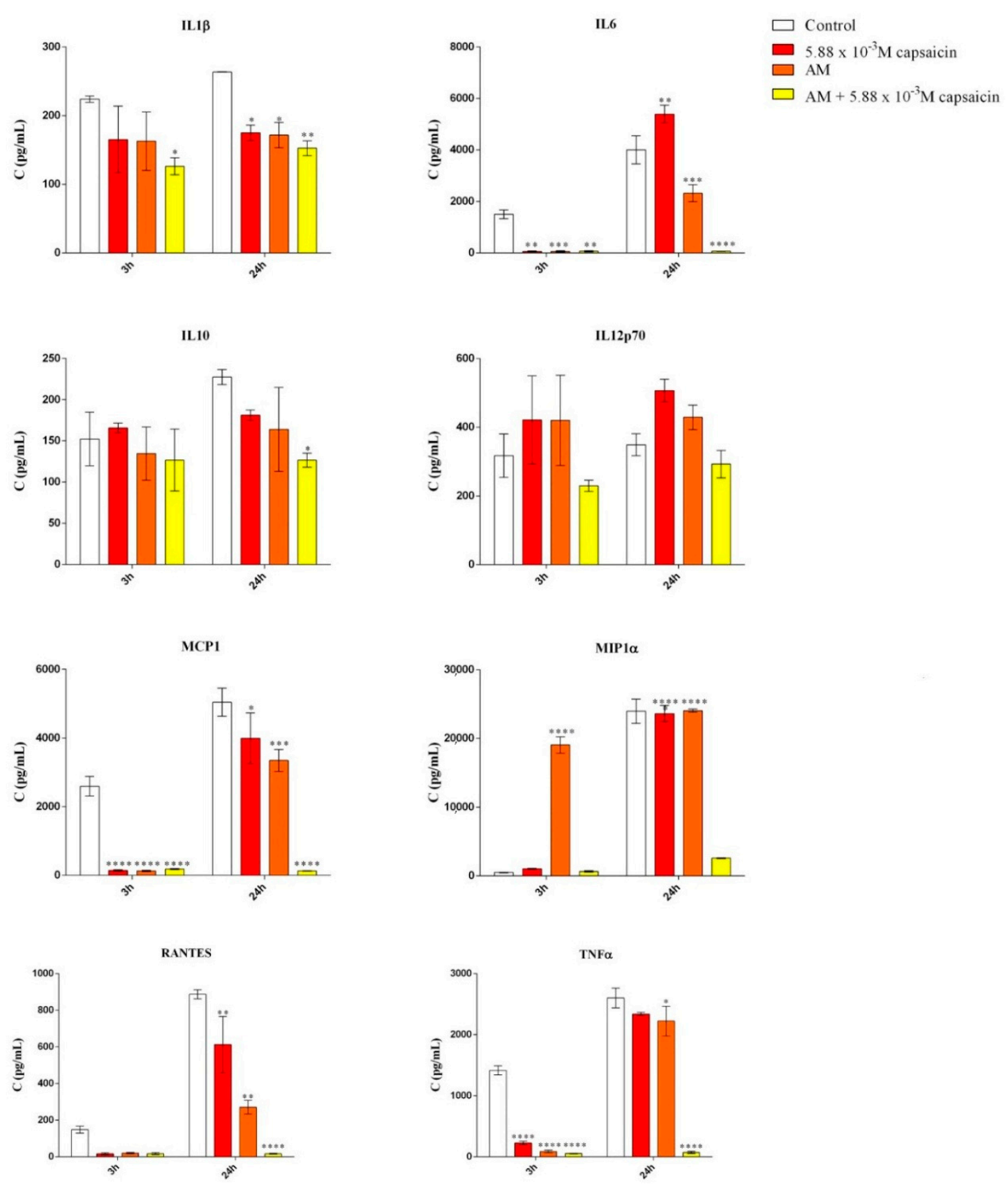

Figure 8. Effects of capsaicin and microencapsulated capsaicin treatment on IL-1 $\beta$, IL-6, IL-10, IL-12p70, MCP-1, MIP-1 $\alpha$, RANTES, and TNF- $\alpha$ production after $3 \mathrm{~h}$ and $24 \mathrm{~h}$ of treatment in LPS-stimulated RAW 264.7 cells $(* p \leq 0.05$ treated vs. control; ${ }^{* *} p \leq 0.01$ treated vs. control; ${ }^{* * *} p \leq 0.001$ treated vs. control; ${ }^{* * *} p \leq 0.0001$ treated vs. control).

\section{Materials and Methods}

\subsection{Alginate Encapsulation of Capsaicin}

Capsaicin, 8-methyl- $N$-vanillyl-6-nonenamide, is a component of a wide variety of red peppers of the genus Capsicum. Its chemical structure is shown in Figure 9.

Capsaicin is a hydrophobic, colorless, odorless, crystalline compound with the molecular formula $\mathrm{C}_{18} \mathrm{H}_{27} \mathrm{NO}_{3}$; its melting point is $62-65^{\circ} \mathrm{C}$, and its molar mass is $305.4 \mathrm{~g} / \mathrm{mol}[4,9]$.

The DrugBank database is a unique bioinformatics and cheminformatics resource that combines detailed drug (i.e., chemical, pharmacological, and pharmaceutical) data with comprehensive drug target (i.e., sequence, structure, and pathway) information. 
<smiles>C=COc1cc(CNC(=O)CCCC/C=C/C(C)C=O)ccc1O</smiles>

Figure 9. The chemical structure of capsaicin (CAS No.: 404-86-4).

Sodium alginate (SA), with a number-averaged molecular weight of $12,000-40,000$, was purchased from Sigma/Merk (Steinheim, Germany) and used as received. Calcium chloride $\left(\mathrm{CaCl}_{2}\right)$ was supplied from Sigma/Merck (Steinheim, Germany) and was used as received. Deionized water was obtained from the lab. All reagents used in this research were obtained as analytical grade.

Alginate solution was prepared by dissolving sodium alginate in distilled water and the solution $(2.5 \%)$ was stirred thoroughly. Stirring was continued after complete addition until a uniform dispersion was obtained. Alginate microcapsules loaded with capsaicin were obtained by ionotropic gelation. The microcapsules were prepared by mixing in the active component (capsaicin extract in ethanol $20 \% \mathrm{~m} / \mathrm{v}$ ) followed by ultrafiltration (calculated after filtration with CHROMAFIL O-45/15 MS filters (Machinery-Nagel GmbH, Germany)), at the concentrations presented in Table 1. The resulting homogenous bubblefree alginate dispersion was extruded using a $21 \mathrm{G}$ syringe needle into the gelation medium, which was kept under stirring to improve the mechanical strength of the beads and to prevent aggregation of the formed beads. The rate of addition was $1.0 \mathrm{~mL} / \mathrm{min}$ at $1100 \mathrm{rpm}$ of stirring speed. The gelation medium was prepared by dispersing different concentrations of calcium chloride solution (5\%).

Table 1. Treatment regimens.

\begin{tabular}{cc}
\hline Treatment & Extracts \\
\hline Alginate microcapsules (AM) & Collected after 30 min, 3 h, and 24 h \\
\hline $1.99 \times 10^{-3}$ M capsaicin-loaded AM & Collected after 30 min, 3 h, and 24 h \\
\hline $2.94 \times 10^{-3}$ M capsaicin-loaded AM & Collected after 30 min, 3 h, and 24 h \\
\hline $5.88 \times 10^{-3}$ M capsaicin-loaded AM & Collected after 30 min, 3 h, and 24 h \\
\hline $1.99 \times 10^{-3}$ M capsaicin & - \\
\hline $2.94 \times 10^{-3}$ M capsaicin & - \\
\hline $5.88 \times 10^{-3}$ M capsaicin & -
\end{tabular}

Encapsulation evaluation has been calculated using Equation (1):

$$
\mathrm{EE}(\%)=\left(\mathrm{P}_{\text {loading }}-\mathrm{P}_{\text {filtration }}\right) / \mathrm{P}_{\text {loading }} \times 100
$$

where $\mathrm{EE}=$ encapsulation efficiency, $\%$; $\mathrm{P}_{\text {loading }}=$ amount of encapsulated capsaicin; $\mathrm{P}_{\text {filtration }}=$ amount of capsaicin in the ultrafiltrate.

A SPECORD M400 (Analytik Jena GmbH, Jena, Germany) and a NOVEX 100 (Novex, Arnhem, The Netherlands) were used for analysis. UV-Vis absorption spectra and the degree of sorption of capsaicin were monitored in solution with a SPECORD M400 spectrophotometer with a monochromator and double beam. Optical microscopy was performed with a NOVEX 100 microscope using the correct magnitude $[18,19]$. 
The microcapsules can be mechanically dispersed by agitation in an aqueous environment; they are sensitive to strong agitation, can be destroyed by large shear forces, and are destroyed by exposure to environments with $\mathrm{pH}$ values lower than 5.5 or greater than 7 . The microcapsules prepared in this manner were maintained for $30 \mathrm{~min}$ in the gelling bath with stirring, and then filtered, washed with distilled water, and dried in an oven at $40^{\circ} \mathrm{C}$.

\subsection{Cell Culture Models and Treatments}

The CCD-1070Sk human dermal fibroblast cell line $\left(\mathrm{ATCC}^{\circledR}\right.$ CRL-2091 $\left.{ }^{\mathrm{TM}}\right)$ was used as an in vitro model for cytotoxicity investigations, as the proposed formulation is intended for skin topical application, while the RAW 264.7 mouse monocyte macrophage cell line (ATCC $^{\circledR}$ TIB-71 ${ }^{\mathrm{TM}}$, Manassas, VA, USA) was used to model in vitro inflammatory status. Both cell lines were purchased from the American Type Culture Collection (ATCC). CCD1070Sk and RAW 264.7 cells were cultured in Eagle's Minimum Essential Medium (MEM) and Dulbecco's Modified Eagle Medium (DMEM), respectively. Both media were supplemented with $10 \%$ fetal bovine serum (FBS) and $1 \%$ antibiotic-antimycotic solution (ABAM, containing $100 \mathrm{U} / \mathrm{mL}$ penicillin, $100 \mu \mathrm{g} / \mathrm{mL}$ streptomycin, and $0.25 \mu \mathrm{g}$ amphotericin B). Both cell lines were sub-cultured weekly and maintained at $37{ }^{\circ} \mathrm{C}$ in a humidified air atmosphere of $5 \% \mathrm{CO}_{2}$ all throughout this study. Media renewal was carried out every other day.

Unloaded alginate microcapsules (AM) and alginate microcapsules loaded with different concentrations of capsaicin (Table 1) were washed with phosphate-buffered saline (PBS) supplemented with 10\% ABAM solution for sterilization purposes, and then immersed in complete culture media for $24 \mathrm{~h}$. After $30 \mathrm{~min}, 3 \mathrm{~h}$, and $24 \mathrm{~h}$, media samples were collected (henceforth referred to as extracts). Collected extracts were stored at $-20^{\circ} \mathrm{C}$ until use. Different capsaicin solutions were freshly prepared in complete culture media and sterilized via $0.22 \mu \mathrm{m}$ filtration, as presented in Table 1.

\subsection{Cytotoxicity Assays}

CCD-1070Sk cells were seeded in 96-well culture plates in triplicate at a final density of $2.5 \times 10^{4}$ cells $/ \mathrm{cm}^{2}$, or in 12-well culture plates under the same conditions for microscopy investigation. After $24 \mathrm{~h}$ of incubation, the culture media was discarded and replaced with the appropriate treatments (shown in Table 1). For experimental controls, the media culture was refreshed at the time of treatment. Untreated samples were used as a reference and were prepared under identical conditions for each assay.

Cell viability was investigated using the 3-(4,5-dimethilthiazol-2-il)-2,5-dipheniltetrazolium bromide (MTT) reduction assay following $24 \mathrm{~h}$ of exposure to treatments [19]. Briefly, the cell medium was replaced with $1 \mathrm{mg} / \mathrm{mL}$ of freshly prepared MTT solution and incubated at $37^{\circ} \mathrm{C}$ for $4 \mathrm{~h}$. Subsequently, the formed formazan crystals were solubilized in 2-propanol and the absorbance was read at $550 \mathrm{~nm}$ using a Flex Station III microplate reader (Molecular Devices, San Jose, CA, USA).

The cytotoxic potential of the screened treatments on CCD-1070Sk dermal fibroblast cells was investigated by the spectrophotometric evaluation of lactate dehydrogenase (LDH) activity in the culture media. Therefore, following $24 \mathrm{~h}$ of exposure to treatments, the culture medium was harvested and mixed with the components of the TOX-7 kit (LDH-Based In Vitro Toxicology Assay Kit, Steinheim, Germany) according to the manufacturer's instructions. After $30 \mathrm{~min}$ incubation at room temperature in the dark, the absorbance of the samples was determined at $490 \mathrm{~nm}$ using a Flex Station III microplate reader (Molecular Devices).

A live/dead fluorescence assay was employed to image cells under treatment conditions. Briefly, CCD-1070Sk cells were stained with a two-color dye solution containing calcein AM (green) and ethidium bromide (red), freshly prepared according to the instructions provided by the manufacturers, in order to highlight live and dead cells at the same time. CCD-1070Sk cells were then incubated at room temperature in the dark for $20 \mathrm{~min}$ with the staining solution and imaged after PBS washing using an Olympus IX73 inverted 
fluorescence microscope. Images were captured using the CellSense imaging software (Olympus, Tokyo, Japan).

\subsection{Cell Morphology Evaluation}

The morphological changes induced by free capsaicin and microencapsulated capsaicin in CCD-1070Sk cell cultures were evaluated by the fluorescent labelling of F-actin filaments. CCD-1070Sk cells were seeded in 12-well culture plates at a final density of $2.5 \times 10^{4}$ cells $/ \mathrm{cm}^{2}$ and incubated for $24 \mathrm{~h}$ with $\mathrm{AM}, 5.88 \times 10^{-3} \mathrm{M}$ capsaicin-loaded $\mathrm{AM}$ extracts collected at $24 \mathrm{~h}$, and $5.88 \times 10^{-3} \mathrm{M}$ capsaicin solution. After exposure, the test media was discarded and the monolayers were washed with PBS, fixed with $4 \%$ paraformaldehyde for $20 \mathrm{~min}$, and permeabilized with $0.1 \%$ Triton X-100/2\% bovine serum albumin for $1 \mathrm{~h}$. Next, the samples were incubated for $1 \mathrm{~h}$ with Alexa Fluor 488-phalloidin (Thermo Fischer Scientific) at $37^{\circ} \mathrm{C}$ to label the actin filaments, and nuclei were counterstained with $2 \mu \mathrm{g} / \mathrm{mL} 4^{\prime}$,6-diamidino-2-phenylindole dihydrochloride (DAPI; Sigma Aldrich) for $15 \mathrm{~min}$. The samples were then inspected by fluorescence microscopy using an Olympus IX73 inverted microscope. Image capturing was performed using CellSense software (Olympus).

\subsection{Reactive Oxygen Species (ROS) Assessment}

Reactive oxygen species (ROS) production was measured by the ROS- $\mathrm{Glo}_{2} \mathrm{O}_{2}$ assay (Promega, Madison, WI, USA), which quantifies the level of hydrogen peroxide released in the culture medium. Briefly, $9.5 \times 10^{4}$ cells/well were seeded in a 12-well culture plate and treated with the collected extracts and different concentrations of capsaicin. For the final $6 \mathrm{~h}$ of treatment, $\mathrm{H}_{2} \mathrm{O}_{2}$ substrate was added at a final concentration of $25 \mu \mathrm{M}$ and incubated at $37^{\circ} \mathrm{C}$ in a humidified atmosphere of $5 \% \mathrm{CO}_{2}$. After $6 \mathrm{~h}$ and $24 \mathrm{~h}$ of treatment, $100 \mu \mathrm{L}$ of ROS-Glo Detection Solution was added, and the plate was incubated for a further $20 \mathrm{~min}$ at room temperature. Finally, luminescence was determined with a Flex Station III microplate reader (Molecular Devices).

\subsection{Inflammatory Status Investigation}

RAW 264.7 cells were seeded in 96-well culture plates at a final density of $2.5 \times 10^{4}$ cells $/ \mathrm{cm}^{2}$ in triplicate. After $24 \mathrm{~h}$ of incubation, the culture media was discarded and replaced with AM, $5.88 \times 10^{-3} \mathrm{M}$ capsaicin loaded AM extracts collected at $24 \mathrm{~h}$, and $5.88 \times 10^{-3} \mathrm{M}$ capsaicin solution. Simultaneously, cells were stimulated with lipopolysaccharide (LPS, $10 \mu \mathrm{g} / \mathrm{mL}$ ). For the experimental controls, the culture media was refreshed, and cells were also stimulated with LPS. After $2 \mathrm{~h}, 6 \mathrm{~h}$, and $24 \mathrm{~h}$, the culture medium was harvested and stored at $-20^{\circ} \mathrm{C}$ until use.

The collected culture media was further used to quantify the nitric oxide (NO) concentration by a method described by Griess [20] using Griess reagent (Promega). Firstly, $50 \mu \mathrm{L}$ of culture supernatant was mixed with $50 \mu \mathrm{L}$ sulfanilamide solution and incubated at room temperature in the dark for $20 \mathrm{~min}$. After $10 \mathrm{~min}$ of incubation, $50 \mu \mathrm{L}$ of N-1napthylethylenediamine dihydrochloride (NED) solution was added. The optical density of the resulting solution was read at $550 \mathrm{~nm}$ using a Flex Station III microplate reader (Molecular Devices). The concentration of $\mathrm{NO}$ was extrapolated from a nitrite standard reference curve that was prepared according to the instructions provided by the manufacturer.

In order to evaluate the inflammatory status of RAW 264.7 cells after treatment, the expression of a panel of cytokines was assessed using a custom Mouse Cytokine/Chemokine Magnetic Bead Panel 96-well Plate Assay (MCYTOMAG-70k; Merck Millipore, Steinhein, Germany). Concentrations of interleukin $1 \beta$ (IL-1 $\beta$ ); interleukin 6 (IL-6); interleukin 10 (IL-10); interleukin 12p70 (IL-12p70); monocyte chemoattractant protein-1 (MCP-1); macrophage inflammatory protein $1 \alpha(\mathrm{MIP} 1 \alpha)$; regulated on activation, normal $\mathrm{T}$ cell expressed, and secreted (RANTES); and tumor necrosis factor $\alpha$ (TNF- $\alpha$ ) were measured using the multiplex magnetic bead panel kit. Deposited aliquots $(25 \mu \mathrm{L})$ of cell culture medium were incubated with anti-cytokine or anti-chemokine antibody-immobilized beads, 
detection antibodies, and streptavidin-phycoerythrin according to the manufacturer's instructions. If needed, samples were adequately diluted in order to fit the linear portion of the standard curve. The plate was analyzed using a MAGPIX reader equipped with xPONENT software (Sigma/Merk, Steinheim, Germany). Standards and quality controls were assayed in duplicate as recommended by the manufacturer. The obtained data were analyzed using MILLIPLEX analysis software (Sigma/Merck, Steinheim, Germany).

\section{Conclusions}

In this study, we showed that our proposed capsaicin encapsulation system alleviates the compound's cytotoxicity, as human dermal fibroblasts showed increased cell viability and decreased LDH activity after $24 \mathrm{~h}$ of treatment with capsaicin-loaded AM compared to control cells. Oxidative stress evaluation proved that the alginate microcapsules and capsaicin-loaded microcapsules were not able to trigger $\mathrm{H}_{2} \mathrm{O}_{2}$ production in CCD-1070Sk cell cultures. Furthermore, using a macrophage cell line for in vitro modeling of an inflammatory environment, we showed that the capsaicin-loaded AM extract significantly decreased NO production compared to the control, while treatment with the same extracts from the encapsulated capsaicin revealed a significand decrease in IL-6 expression compared to the controls. Similar patterns of expression were observed for MCP-1, MIP-1 $\alpha$, RANTES, and TNF- $\alpha$.

Author Contributions: Formal analysis, A.H., B.G. and L.I.; Investigation, A.H., B.G. and L.I.; Methodology, A.H., B.G., M.C., R.-M.I. and O.G.; Project administration, C.N. and O.G.; Writingoriginal draft, A.H., B.G. and R.-M.I.; Writing—review and editing, M.C., O.G. and C.N. All authors have read and agreed to the published version of the manuscript.

Funding: This research was financed by the UEFISCDI, through the BG120/01/10/2016-NANOCAPTOX grant.

Data Availability Statement: The data presented in this study are available on request from the corresponding author.

Conflicts of Interest: The authors declare no conflict of interest. The funders had no role in the design of the study; in the collection, analyses, or interpretation of data; in the writing of the manuscript, or in the decision to publish the results.

Sample Availability: Samples of the compounds are not available from the authors.

\section{References}

1. Schumacher, M. TRP channels in pain and inflammation: Therapeutic opportunities. Pain Pract. 2010, 10, 185-200. [CrossRef] [PubMed]

2. Turnbull, A. Tincture of Capsaicin as a Remedy for Chilblains and Toothache. Dublin Free Press 1850, 1, 95-96.

3. Vriens, J.; Nilius, B.; Vennekens, R. Herbal compounds and toxins modulating TRP channels. Curr. Neuropharmacol. 2008, 6, 79-96. [PubMed]

4. Reyes-Escogido, M.d.L.; Gonzalez-Mondragon, E.; Vazquez-Tzompantzi, E.G. Chemical and pharmacological aspects of capsaicin. Molecules 2011, 16, 1253-1270. [CrossRef] [PubMed]

5. Ginghina, O.; Negrei, C.; Hudita, A.; Ioana-Lavric, I.; Galateanu, B.; Dragomir, S.; Burcea Dragomiroiu, G.T.A.; Barca, M.; Nitipir, C.; Diaconu, C.C.; et al. In Vitro Impact of Some Natural Compounds on Ht-29 Colorectal Adenocarcinoma Cells. Farmacia 2017, 65, 947-953.

6. Winter, J.; Bevan, S.; Campbell, E. Capsaicin and pain mechanisms. Br. J. Anaesth. 1995, 75, 157-168. [CrossRef] [PubMed]

7. Knotkova, H.; Pappagallo, M.; Szallasi, A. Capsaicin (TRPV1 Agonist) therapy for pain relief farewell or revival? Clin. J. Pain. 2008, 24, 142-154. [CrossRef] [PubMed]

8. Anand, P.; Bley, K. Topical capsaicin for pain management: Therapeutic potential and mechanisms of action of the new highconcentration capsaicin 8\% patch. Br. J. Anaesth. 2011, 107, 490-502. [CrossRef] [PubMed]

9. Hayman, M.; Kam, P.C.A. Capsaicin: A review of its pharmacology and clinical applications. Curr. Anaesth. Crit. Care 2008, 19, 338-343. [CrossRef]

10. Gooding, S.M.; Canter, P.H.; Coelho, H.F.; Boddy, K.; Ernst, E. Systematic review of topical capsaicin in the treatment of pruritus. Int. J. Dermatol. 2010, 49, 858-865. [CrossRef] [PubMed] 
11. Ilie, M.A.; Caruntu, C.; Tampa, M.; Georgescu, S.R.; Matei, C.; Negrei, C.; Ion, R.M.; Constantin, C.; Neagu, M.; Boda, D. Capsaicin: Physicochemical properties, cutaneous reactions and potential applications in painful and inflammatory conditions. Exp. Ther. Med. 2019, 18, 916-925. [CrossRef] [PubMed]

12. Casanueva, B.; Rodero, B.; Quintial, C.; Llorca, J.; Gonzalez-Gay, M.A. Short- term efficacy of topical capsaicin therapy in severely affected fibromyalgia patients. Rheumatol. Int. 2013, 33, 2665-2670. [CrossRef] [PubMed]

13. Simpson, D.M.; Brown, S.; Tobias, J.K.; Vanhove, G.F. NGX-4010, a capsaicin $8 \%$ dermal patch, for the treatment of painful HIV-associated distal sensory polyneuropathy: Results of a 52-week open-label study. Clin. J. Pain. 2014, 30, 134-142. [CrossRef] [PubMed]

14. Ren, K.; Torres, R. Role of interleukin-1 $\beta$ during pain and inflammation. Brain Res. Rev. 2009, 60, 57-64. [CrossRef] [PubMed]

15. Lee, K.M.; Jeon, S.M.; Cho, H.J. Interleukin-6 induces microglial CX3CR1 expression in the spinal cord after peripheral nerve injury through the activation of p38 MAPK. Eur. J. Pain. 2010, 14, 682. [CrossRef] [PubMed]

16. Kiguchi, N.; Maeda, T.; Kobayashi, Y.; Fukazawa, Y.; Kishioka, S. Macrophage inflammatory protein-1alpha mediates the development of neuropathic pain following peripheral nerve injury through interleukin-1beta up-regulation. Pain 2010, 149, 305-315. [CrossRef] [PubMed]

17. Seifert, F.; Maihöfner, C. Functional and structural imaging of pain-induced neuroplasticity. Curr. Opin. Anaesthesiol. 2011, 24, 515-523. [CrossRef] [PubMed]

18. Paques, J.P.; van der Linden, E.; van Rijn, C.J.; Sagis, L.M. Preparation methods of alginate nanoparticles. Adv. Colloid. Interface Sci. 2014, 209, 163-171. [CrossRef] [PubMed]

19. Lee, K.Y.; Mooney, D.J. Alginate: Properties and biomedical applications. Prog. Polym. Sci. 2012, 37, 106-126. [CrossRef] [PubMed]

20. Griess, P.; Bemerkungen zu der abhandlung der, H.H. Weselsky und Benedikt "Ueber einige azoverbindungen". Chem. Ber. 1879, $12,426-428$. 